\title{
Kinetics of dissolution of glass fibre in hot alkaline solution
}

\author{
S. T. Bashir ${ }^{1}$, L. Yang ${ }^{1, *}$ (D) J. J. Liggat ${ }^{2}$, and J. L. Thomason ${ }^{1}$ \\ ${ }^{1}$ Department of Mechanical and Aerospace Engineering, University of Strathclyde, 75 Montrose Street, Glasgow G1 1XJ, UK \\ ${ }^{2}$ Department of Pure and Applied Chemistry, University of Strathclyde, 295 Cathedral Street, Glasgow G1 1XL, UK
}

Received: 3 July 2017

Accepted: 20 September 2017

Published online:

25 September 2017

(C) The Author(s) 2017. This article is an open access publication

\begin{abstract}
Kinetics of the dissolution of E-glass fibres in alkaline solutions was investigated. To allow an accurate determination of conversion, glass fibres were immersed individually in the corrosive medium and the diameter change was measured with the use of a scanning electron microscope. Few studies have been reported in the literature on the kinetics of E-glass fibre dissolution or the dissolution of individual fibres. Our experimental results fit well in the zeroorder and shrinking cylinder models, suggesting either the diffusion of hydroxide ions through the solution or the glass fibre etching itself was ratelimiting step. The rate constant for the reaction of glass fibre with alkaline solution at $95{ }^{\circ} \mathrm{C}$ was found to be between $1.3 \times 10^{-4}$ and $4.3 \times 10^{-4} \mathrm{~g} /\left(\mathrm{m}^{2} \mathrm{~s}\right)$. The reaction order $(n)$ was determined as $0.31-0.49$ with respect to the alkaline solution, and the activation energy was $58-79 \mathrm{~kJ} / \mathrm{mol}$.
\end{abstract}

\section{Introduction}

Glass fibre is a vital reinforcement material in various composite applications. It is estimated that by 2020 the composites market will reach almost $£ 80$ billion globally [1]. Glass fibre, usually of E-glass composition, is currently used as reinforcement in over $90 \%$ of all fibre-reinforced composites produced [2]. The high mechanical properties and chemical resistance of these composites, particularly glass fibre-reinforced thermosetting polymers, are required for optimum performance but unfortunately result in poor recyclability. When such materials are no longer fit for purpose, they are deposited frequently through landfilling. The rising costs associated with landfill together with increasingly stringent legislation mean this disposal route is becoming ever more undesirable. Consequently, alternative methods for dealing with composite manufacturing waste are needed [3]. In addition, the accelerating growth in the use of glass fibre-reinforced plastics such as in the production of wind turbine blades [4] and in the construction sector means it is imperative that a long-term, cost-effective, recycling solution be developed for end-of-life composites.

In an effort to reduce the environmental damage caused by disposal of end-of-life composite materials, a range of recycling techniques have been investigated, some of which are now exploited on an industrial scale $[5,6]$. Thermal treatment is one of the

Address correspondence to E-mail: 1.yang@strath.ac.uk 
most widespread recycling technologies; by subjecting the composite to elevated temperatures, degradation of the polymeric matrix is achieved, facilitating subsequent extraction of any fibrous reinforcement. Due to the harsh conditions employed in this procedure, the glass fibres suffer from a severe loss in their ultimate strength and therefore cannot be reused in many forms of composite applications [7-10]. We have recently developed a simple chemical approach to address this key issue and have successfully proved that these damaged filaments can be reused as reinforcements again if their strength is restored [11]. It was found that the strength of glass fibres heat-conditioned at 450-600 ${ }^{\circ} \mathrm{C}$ can almost triple after a few minutes of immersion in dilute hydrofluoric acid (HF) [12]. HF is proven to be an effective chemical etchant and is thought to strengthen glass by removing surface flaws [13]. In fact, the reduction in fibre diameter after HF treatment can be related to strength increase [12]. Due to the toxicity of HF, it cannot be easily implemented on a commercial scale for regenerating strength of thermally weakened glass fibres. However, we have recently discovered that hot alkaline solution, such as sodium hydroxide $(\mathrm{NaOH})$ and potassium hydroxide $(\mathrm{KOH})$, can improve the strength of thermally degraded glass fibres in a similar manner to HF [14, 15]. The dissolution of silicate glass in alkali is well documented in literature [16-18]; however, the use of these corrosive substances for strengthening thermally damaged glass fibres is a novel concept; it is believed the reaction of silica $\left(\mathrm{SiO}_{2}\right)$ with hydroxide ions $\left(\mathrm{OH}^{-}\right)$from the alkaline solution [19] leads to a modification of the damaged fibre surface and improves tensile strength. We have found that the strength increase from a short hot alkaline treatment can be achieved at little expense of removing surface materials [11]. This contrasts with the behaviour of HF, which significantly etches the glass fibre surface at its optimum treatment duration of $2.5 \mathrm{~min}$ [12]. Understanding and controlling the strength regeneration mechanism of thermally damaged glass fibres through alkaline treatments requires the investigation of the kinetics of the reaction between the glass and the alkaline solution. To obtain accurate and reliable kinetic data, the conversion of glass fibres after alkaline treatment needs to be measurable. Although an excessive alkaline treatment can eventually lead to strength decrease of thermally damaged fibres, a measurable fibre diameter reduction can be obtained, particularly when fibres are immersed individually in the alkaline solution.

Dissolution experiments are mainly conducted with bulk silicate glasses in the literature, and glasses which include a significant amount of various metal oxides in their formulation, such as E-glass, are not explored very often. In addition, little study on the dissolution kinetics of glass fibres immersed individually in alkaline solution has been conducted. Our present investigation focuses on the reaction kinetics of E-glass fibre dissolution in alkaline solution. Individual E-glass fibres were immersed in the corrosive medium for prolonged periods of time, rinsed to remove residue and measured for obtaining fibre diameter with a scanning electron microscope (SEM). Measurement was taken of the corresponding untreated fibre in order to calculate the fibre diameter reduction. It is thought the effect of etching of the glass surface by alkaline treatment is strongly dependent on various reaction conditions including nature of alkaline solution, temperature, concentration and treatment duration. Both $\mathrm{KOH}$ and $\mathrm{NaOH}$ were investigated as a corrosive medium in this work. The temperature and concentration of these alkaline solutions and treatment duration were varied to determine the activation energy and reaction order, respectively.

\section{Experimental}

\section{Materials}

Boron-free E-glass fibres supplied by Owens Corning (OC) were used in this study. These OC fibre rovings were manufactured on a pilot scale bushing and received as $20-\mathrm{kg}$ continuous single-end square-edge packages. Each roving had a nominal tex of 1200 and a nominal fibre diameter of $17 \mu \mathrm{m}$. The fibres were sprayed with water after production; these fibres are therefore commonly referred to as 'water-sized' or 'bare'. Mechanical properties of these fibres at roomtemperature are reported elsewhere [20]. The chemicals used in this project were purchased from VWR and included $\mathrm{NaOH}$ pellets, $\mathrm{KOH}$ flakes (both at commercial grade) and standard $37 \%$ concentrated hydrochloric acid $(\mathrm{HCl})$. 


\section{Single fibre treatment in alkaline solution}

Glass fibres were treated individually in alkaline solution whilst varying particular reaction parameters such as temperature and concentration of solution and treatment time. Figure 1 shows a schematic of the fibre sample preparation for alkaline treatment. Each fibre was cut into three portions: the first portions were the control (untreated) fibres and the other two portions were the fibres to be treated in $\mathrm{KOH}$ and $\mathrm{NaOH}$ solution at a particular set of reaction conditions which involved varying the conditions of alkaline solution as indicated in Table 1. Five fibre samples were prepared for the treatment at each time. The fibres were mounted on labelled cards with tape, and the length of fibre immersed in alkaline solution was around $3 \mathrm{~cm}$.

$\mathrm{NaOH}$ and $\mathrm{KOH}$ solutions were prepared in polypropylene containers according to the following concentrations: $0.5,1.0,1.5,2.0$ and $3.0 \mathrm{~mol} / \mathrm{L}$. Five hundred millilitres of solution was prepared for each treatment to fully immerse the fibres. Containers were sealed and heated to $95{ }^{\circ} \mathrm{C}$ before treating the fibre samples. The fibres were immersed in solution by mounting the cards on the inside of the container. After each treatment time, the designated fibre

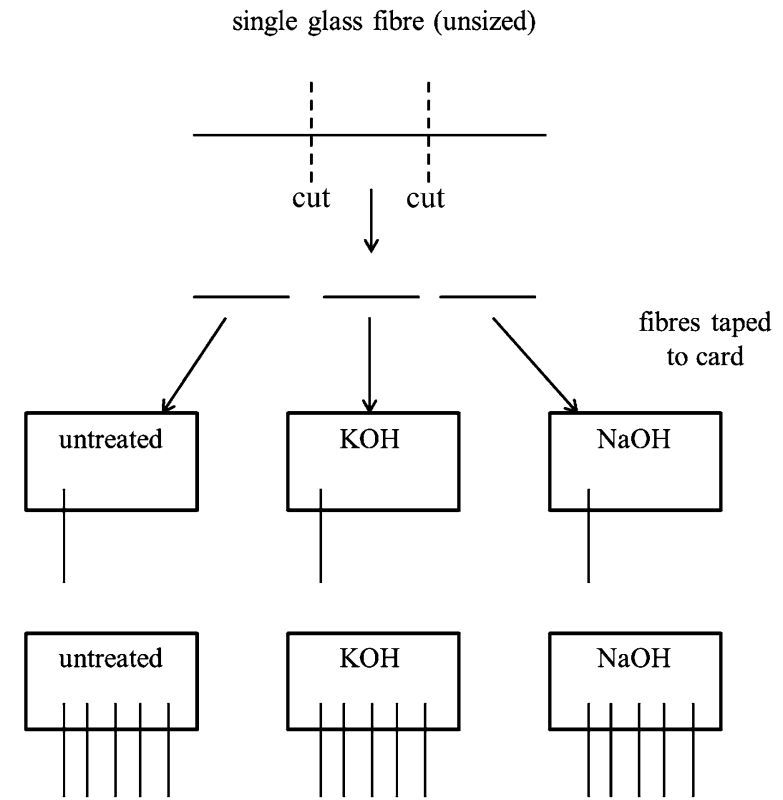

5 fibres treated in $\mathrm{KOH}$ and $\mathrm{NaOH}$ solution at particular concentration and temperature for 1,2, 3 and 5 hours, acid-rinsed and dried

Figure 1 Schematic showing the fibre sample preparation for alkaline treatment. samples were removed from the solution, rinsed in diluted $\mathrm{HCl}(10 \% \mathrm{v} / \mathrm{v})$ for $10 \mathrm{~min}$, followed by rinsing with deionised water for $1 \mathrm{~min}$. The purpose of this rinsing procedure, particularly with acid, was to ensure the effective removal of any residual deposits which could have remained on the fibre surface at the end of the alkaline treatment [21]. In addition, previous experimentation did not show any significant change in glass fibre diameter after this acid rinsing procedure alone. Once the fibres were rinsed after alkaline treatment, they were left to dry in an oven at $110{ }^{\circ} \mathrm{C}$ for $15 \mathrm{~min}$. By varying the concentration of solution $(0.5-3.0 \mathrm{~mol} / \mathrm{L})$ at a constant temperature of $95^{\circ} \mathrm{C}$, the kinetic data can allow the determination of the reaction order with respect to $\mathrm{KOH}$ and $\mathrm{NaOH}$. Furthermore, the variation of solution temperature $\left(75,80,85,90\right.$ and $\left.95{ }^{\circ} \mathrm{C}\right)$ at a constant concentration of $3 \mathrm{~mol} / \mathrm{L}$ means the activation energy of the reaction of glass fibre with alkaline solution can be calculated. After alkaline treatment, fibres were mounted for measurement under SEM, and the fibre diameter reduction was determined for each reaction condition using the following equation:

$\Delta D=\frac{D_{\mathrm{o}}-D_{\mathrm{t}}}{D_{\mathrm{o}}}$

$D_{\mathrm{o}}$ is the initial fibre diameter (untreated) and $D_{\mathrm{t}}$ is the diameter after treatment of the fibre in alkaline solution for time $t$. The diameters of around five different fibres were measured, and for each fibre, measurements of diameter were made at three different points (although it was found that the diameter was consistent throughout the length of the fibre). The diameters of each fibre were then averaged to give the average fibre diameter reduction for a particular reaction condition (for example, $3 \mathrm{M} \mathrm{NaOH}$, $\left.95^{\circ} \mathrm{C}, 1 \mathrm{~h}\right)$.

\section{Scanning electron microscopy (SEM)}

A HITACHI SU-6600 field emission scanning electron microscope (FE-SEM) was used for surface morphology analysis of fibres and to measure diameters after alkaline treatment. Samples were coated in gold using an Edwards S150 sputter coater in order to prevent charge build-up as glass fibres are non-conductive. The gold coating is normally a few nanometres thick and is negligible with respect to the fibre diameter change observed in this work. The electron beam was rotated so that the fibre was as 
Table 1 Alkaline treatment conditions employed for kinetic study of glass fibre dissolution

\begin{tabular}{llll}
\hline Condition & Concentration of alkaline solution $(\mathrm{mol} / \mathrm{L})$ & Temperature of alkaline solution $\left({ }^{\circ} \mathrm{C}\right)$ & Treatment time in alkaline solution $(\mathrm{h})$ \\
\hline 1 & 0.5 & 95 & $1,2,3,5$ \\
2 & 1.0 & 95 & $1,2,3,5$ \\
3 & 1.5 & 95 & $1,2,3,5$ \\
4 & 2.0 & 95 & $1,2,3,5$ \\
5 & 3.0 & 95 & $1,2,3,5$ \\
6 & 3.0 & 75 & $1,2,3,5$ \\
7 & 3.0 & 80 & $1,2,3,5$ \\
8 & 3.0 & 85 & $1,2,3,5$ \\
9 & 3.0 & 90 & $1,2,3,5$ \\
\hline
\end{tabular}

horizontal as possible in order to measure fibre diameter through automatic scaling of the SEM. SEM images were captured at an accelerating voltage of $15 \mathrm{kV}$ and extraction voltage of $1.8 \mathrm{kV}$.

\section{Results and discussion}

\section{Effect of alkaline solution concentration on diameter reduction in glass fibres}

Figure 2 shows the fibre diameter reduction in the glass fibres following treatment in $\mathrm{KOH}$ solution at $0.5,1.0,1.5,2.0$ and $3.0 \mathrm{~mol} / \mathrm{L}$ for $1,2,3$ and $5 \mathrm{~h}$, with a constant $\mathrm{KOH}$ solution temperature of $95{ }^{\circ} \mathrm{C}$. The error bars used for measurements of diameter reduction show 95\% confidence limits. From Fig. 2, it is clear that for each $\mathrm{KOH}$ solution concentration the fibre diameter reduction increases at a constant rate with time, though in some cases there seems to be an induction period before this linear increase. In addition, the diameter reduction for each treatment time

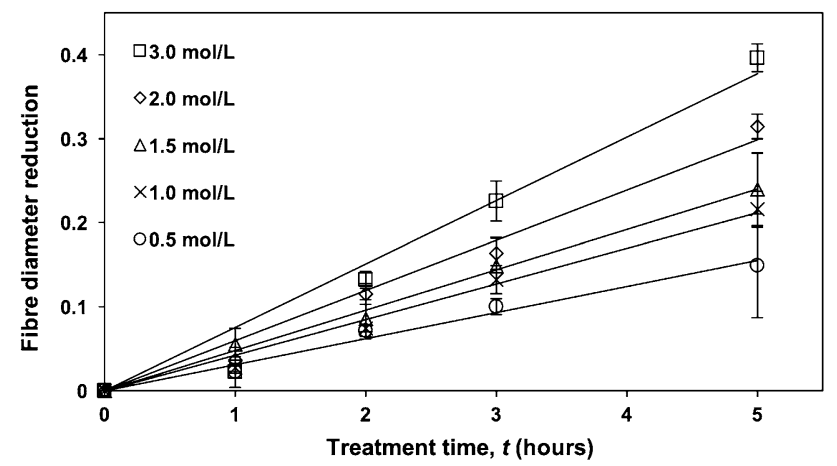

Figure 2 Diameter reduction in glass fibres after treatment in $\mathrm{KOH}$ solution at various concentrations and times. appears to increase with $\mathrm{KOH}$ solution concentration. The fact that the diameter reduction increases at a constant rate with time means the slope of the line can be used to denote the rate of fibre diameter reduction.

Figure 3 shows the fibre diameter reduction with time for fibres treated in $\mathrm{NaOH}$ at various concentrations. It presents overall a similar trend to that for $\mathrm{KOH}$ in Fig. 2 as the diameter reduction increases linearly with time, and the rate increases as a function of concentration. The glass dissolution process in hot $\mathrm{KOH}$ and $\mathrm{NaOH}$ solutions involves many elementary steps; the hydroxide ions initially have to diffuse through the bulk solution phase, and eventually some of these ions would come into contact with the localised glass surface and potentially react. It is therefore the interaction of the hydroxide ions with the glass surface which results in glass fibre dissolution/diameter reduction. From our experimental results, it is clear that the rate of dissolution of the glass fibres increases with solution concentration. This could be due to more hydroxyl ions being able to

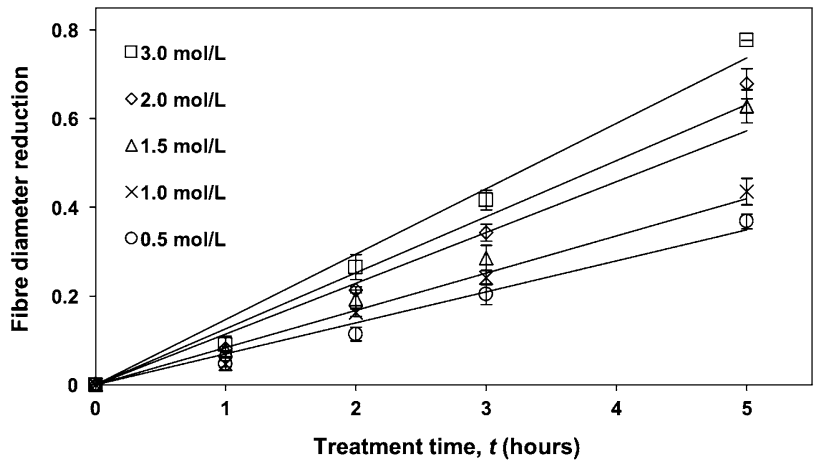

Figure 3 Diameter reduction in glass fibres after treatment in $\mathrm{NaOH}$ solution at various concentrations and times. 
collide with the glass fibre surface with enough energy to result in a reaction, as there was an increased concentration in the bulk phase originally.

Figure 4 summarises the effect of solution concentration of $\mathrm{KOH}$ and $\mathrm{NaOH}$ on the rate of fibre diameter reduction. It can be observed from Fig. 4 that the rate of diameter reduction is linearly correlated with the concentration of both $\mathrm{KOH}$ and $\mathrm{NaOH}$ solutions. In addition, it is found that the diameter reduction rate in $\mathrm{NaOH}$ solution is approximately double that in $\mathrm{KOH}$ solution at a given concentration. This suggests that $\mathrm{NaOH}$ is a more corrosive E-glass fibre etchant than $\mathrm{KOH}$, and this is consistent with previous investigations concerning bulk glass $[16,22,23]$. The disparity in behaviour of $\mathrm{KOH}$ and $\mathrm{NaOH}$ with E-glass fibre is clear in Fig. 5, which shows SEM images of a fibre untreated and after treatment in $3 \mathrm{~mol} / \mathrm{L} \mathrm{KOH}$ and $\mathrm{NaOH}$ for $5 \mathrm{~h}$ at $95{ }^{\circ} \mathrm{C}$.

Generally, the chemical strength of basic hydroxides is determined by their dissociation constants, which indicate their ability to separate into metal

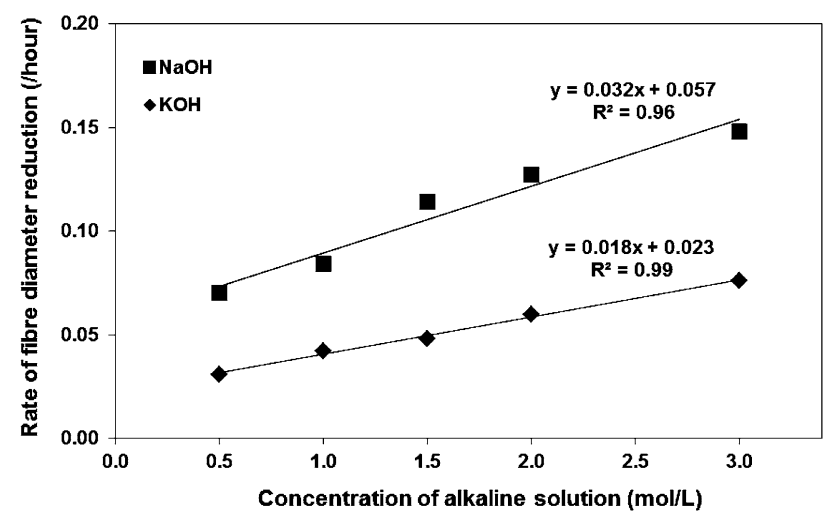

Figure 4 Rate of diameter reduction in glass fibres after treatment in $\mathrm{KOH}$ and $\mathrm{NaOH}$ solution at various concentrations. cations and hydroxide anions in solution. Since potassium has a greater atomic size than sodium, one might assume $\mathrm{KOH}$ to be a stronger base. However, our observations with E-glass fibre and previous studies with bulk glass $[16,22,23]$ show $\mathrm{NaOH}$ is the most corrosive alkali towards glass. The reason for this is seldom explained, though it is suggested it could be due to the reaction products from $\mathrm{NaOH}$ and glass being formed more readily and more exothermally [22]. Another possible theory is that the smaller $\mathrm{Na}^{+}$ions have a stronger attraction to $\mathrm{OH}^{-}$ ions in solution, which reduces the likelihood of $\mathrm{OH}^{-}$ ions to interact with neighbouring water molecules and as a result they are more likely to come into contact with the glass [24].

\section{Effect of alkaline solution temperature on diameter reduction in glass fibres}

Figure 6 displays a plot of the fibre diameter reduction in glass fibres after treatment in $\mathrm{KOH}$ solution for $1,2,3$ and $5 \mathrm{~h}$ at a constant concentration of $3 \mathrm{~mol} / \mathrm{L}$, whilst varying the solution temperature (75, $80,85,90$ and $95^{\circ} \mathrm{C}$ ). For each temperature, it is evident the diameter reduction increases at a constant rate with time. As a result, the gradient from plotting the diameter reduction against time at each temperature can be used as an indication of the rate at which the glass fibre has reacted with the solution. It is clear from the results in Fig. 6 that the reaction rate is increased by raising temperatures.

Figure 7 shows the diameter reduction in glass fibres after treatment in $\mathrm{NaOH}$ solution at $75-95{ }^{\circ} \mathrm{C}$ for $1,2,3$ and $5 \mathrm{~h}$. Similar to the case of $\mathrm{KOH}$ in Fig. 6, we see a constant increase in the fibre diameter reduction with time at a given solution temperature and reaction rate increases as temperature increases. Figure 8 gives the rate of fibre diameter reduction
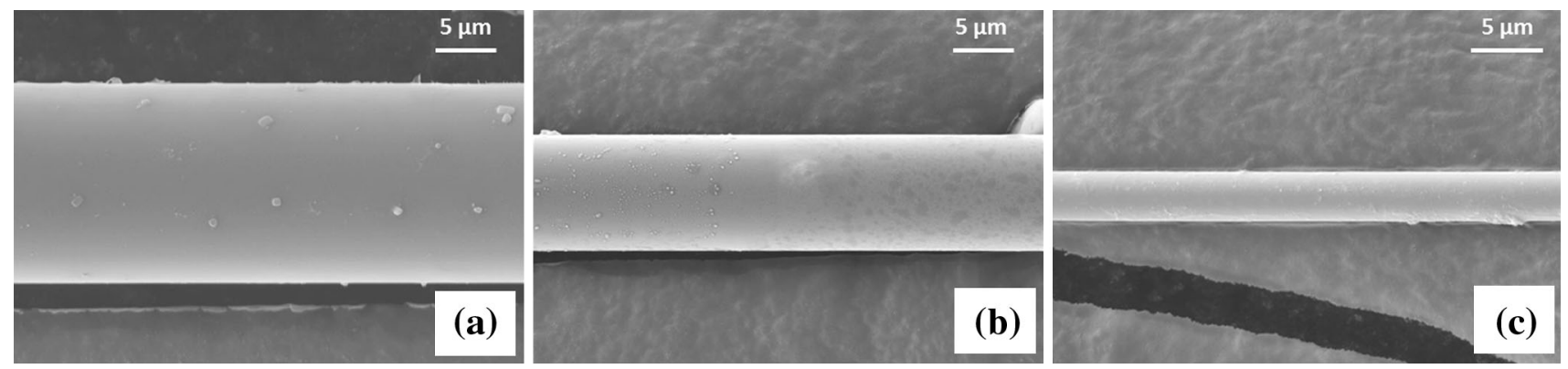

Figure 5 SEM images of glass fibre a untreated $(16.5 \mu \mathrm{m}), \mathbf{b}$ treated in $3 \mathrm{~mol} / \mathrm{L} \mathrm{KOH}$ at $95{ }^{\circ} \mathrm{C}$ for $5 \mathrm{~h}(9.7 \mu \mathrm{m})$ and $\mathbf{c}$ treated in $3 \mathrm{~mol} / \mathrm{L}$ $\mathrm{NaOH}$ at $95{ }^{\circ} \mathrm{C}$ for $5 \mathrm{~h}(3.5 \mu \mathrm{m})$. 


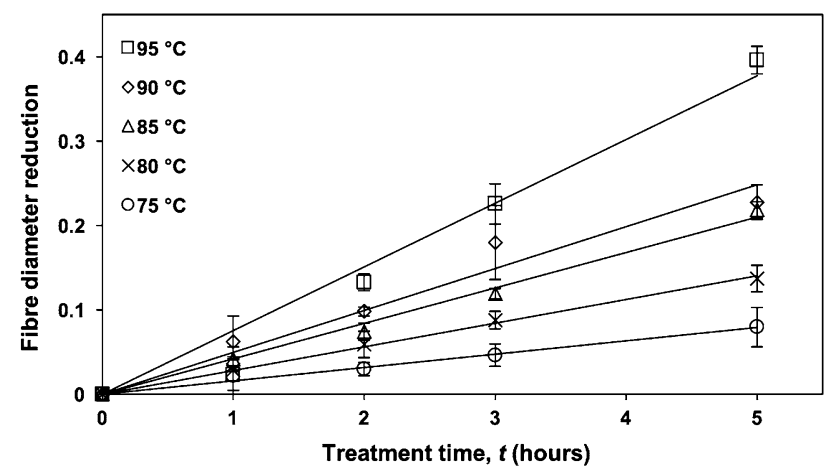

Figure 6 Diameter reduction in glass fibres after treatment in $\mathrm{KOH}$ solution at various temperatures and times.

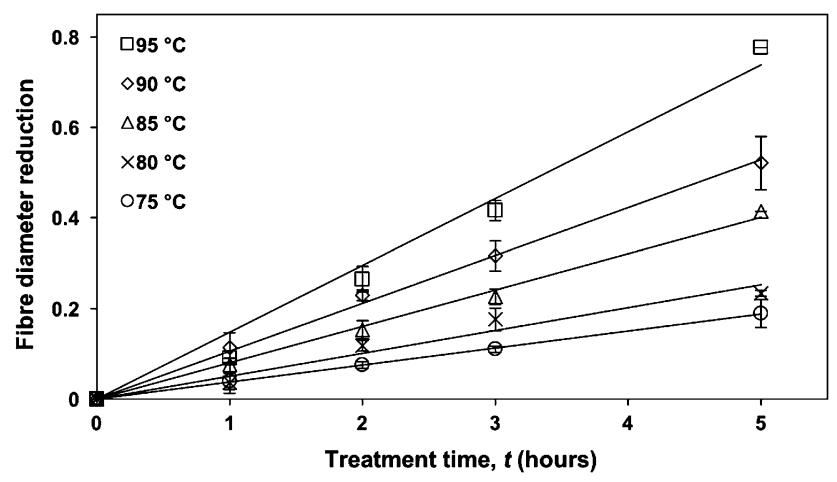

Figure 7 Diameter reduction in glass fibres after treatment in $\mathrm{NaOH}$ solution at various temperatures and times.

against the temperature of $\mathrm{KOH}$ and $\mathrm{NaOH}$ solution. It is noticed that the temperature dependence of the rate of diameter reduction follows an exponential fashion. This comes as no surprise given that the effect of reaction temperature on rate constant is typically described by the Arrhenius equation, which is essentially an exponential function. Again, it appears that $\mathrm{NaOH}$ overall reduces the glass fibre diameter up to twice the magnitude of $\mathrm{KOH}$ for each solution temperature.

In order for a reaction to occur, reactants must not only collide with each other, but the collision needs to possess enough energy to overcome the activation barrier of the reaction; this is referred to as the activation energy $\left(E_{\mathrm{a}}\right)$ and can be determined from the Arrhenius equation. As the temperature increases, the hydroxide anions gain more kinetic energy and therefore there will be statistically more collisions with enough energy to result in a reaction. On the other hand, the level of temperature variation in the present work would have little influence on the

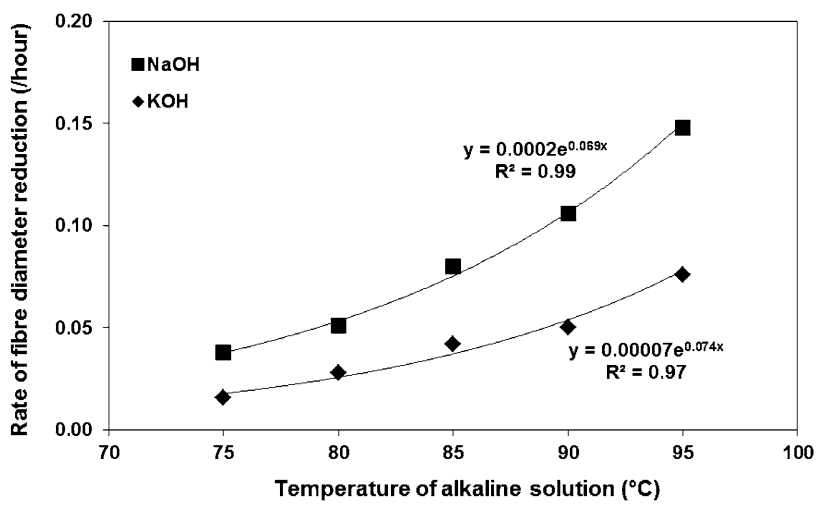

Figure 8 Rate of diameter reduction in glass fibres after treatment in $\mathrm{KOH}$ and $\mathrm{NaOH}$ solution at various temperatures.

kinetic energy of the glass network as it is in the solid state.

\section{Modelling of glass fibre dissolution in alkaline solution}

$\mathrm{SiO}_{2}$ is the major component in virtually all types of glass fibre, with various metal oxides such as $\mathrm{Al}_{2} \mathrm{O}_{3}$, $\mathrm{CaO}$ and $\mathrm{MgO}$ often included in the formulation. For simplicity, we refer to silica glass as $\mathrm{SiO}_{2}$, but in reality the $\mathrm{SiO}_{2}$ exists as a complex network. In addition $\mathrm{Al}_{2} \mathrm{O}_{3}$, classed as an intermediate oxide, can function both as a network former and modifier in the glass. $\mathrm{CaO}$ and $\mathrm{MgO}$ are included in glass as network modifiers only. When glass is in contact with hot, concentrated alkaline solution, the hydroxyl ions attack the $\mathrm{SiO}_{2}$ framework according to the below expression (2). $\mathrm{Al}_{2} \mathrm{O}_{3}$, which is the third largest component in E-glass, can react with $\mathrm{OH}^{-}$from $\mathrm{NaOH}$ and $\mathrm{KOH}$ solution in a similar fashion to $\mathrm{SiO}_{2}$ as shown in Eq. (3):

$[-\mathrm{Si}-\mathrm{O}-\mathrm{Si}-]+\mathrm{OH}^{-} \stackrel{\Delta}{\rightarrow}[-\mathrm{SiO}]^{-}+[-\mathrm{Si}-\mathrm{OH}]$

$[-\mathrm{Al}-\mathrm{O}-\mathrm{Al}-]+\mathrm{OH}^{-} \stackrel{\Delta}{\rightarrow}[-\mathrm{AlO}]^{-}+[-\mathrm{Al}-\mathrm{OH}]$

Due to their basic nature, $\mathrm{CaO}$ (which is the second largest E-glass component) and $\mathrm{MgO}$ may not react directly with $\mathrm{KOH}$ or $\mathrm{NaOH}$ but they are soluble in the alkaline solution, resulting in further disruption of the glass network structure. It is thought that the breakdown of the silicate network is a key process of the reaction of E-glass fibre with alkaline solution, and the dispersion of network modifiers such as $\mathrm{CaO}$ into solution can also contribute to the fibre dissolution and diameter reduction. Gin et al. [25] proposed that ion exchange was the dominant process in the 
reaction of bulk glass with alkaline solution prepared at $\mathrm{pH} 9$ and $90{ }^{\circ} \mathrm{C}$, and at higher concentrations $(\mathrm{pH}$ 11.5) there was a rapid dissolution of the glass due to hydroxide attack. Our solutions were prepared at a $\mathrm{pH}$ above 14, in which case the hydrolysis of the glass network is believed to be a more dominating process of the reaction than ion exchange.

Various models have been developed and are used extensively to describe solid-state reactions [26]. These models require the input of the conversion $(\alpha)$, which is a measurement of the change in mass of the solid before and after the reaction according to Eq. (4):

$\alpha=\frac{M_{\mathrm{o}}-M_{\mathrm{t}}}{M_{\mathrm{o}}}$

$M_{\mathrm{o}}$ is the initial mass of the solid (at $t=0$ ) and $M_{\mathrm{t}}$ is the mass of the remaining solid at time $t$. Usually, the conversion values are extracted directly from mass change but because our study involved the dissolution of individual glass fibres, it was more appropriate to measure the fibre diameter reduction using an SEM and then calculating the conversion using the below expression:

$\alpha=1-(1-\Delta D)^{2}$

where $\Delta D$ is the fibre diameter reduction.

There are three possible rate-determining steps in the reaction of glass with alkaline solution which need to be considered. One of them is the diffusion of $\mathrm{OH}^{-}$ions through the solution and can be described by the zero-order model (6a). This model relates the conversion rate of the glass to the diffusion rate of the ions. The reaction is zero order with respect to the glass; thus, Eq. (6a) is essentially the expression for zero-order kinetics:

$\alpha=r t$

The reaction rate (or conversion rate) $(r)$ can be extracted from the slope of the conversion of the glass fibres $(\alpha)$ against reaction time $(t)$. The conversion rate is dependent on factors such as the glass surface area, concentration of the solution and the diffusion rate of the $\mathrm{OH}^{-}$ions.

Another potential rate-limiting step is the hydroxide attack of the glass fibre surface, which results in a reduction in fibre diameter and can therefore be defined by the shrinking cylinder model (6b):

$1-(1-\alpha)^{1 / 2}=r t$
Here, Eq. (4) is modified to relate the conversion of a cylinder with the change in radius over time, eventually leading to expression (6b); the full mathematical derivation is given elsewhere [26]. Notice how rearranging Eq. (6b) to find $\alpha$ leads to an expression of a similar form to (5) where $r t=\Delta D$, proving that the shrinking cylinder model, in effect, gives the fibre diameter reduction. The reaction rate $r$ can therefore be considered as the rate of fibre diameter reduction, and its value is related to the hydroxide concentration on the glass surface and the glass surface area.

Finally, when the glass reacts with the alkaline solution a product layer may form around the surface, meaning the hydroxide ions need to subsequently diffuse through this layer before reacting with the glass. The product layer thickness can increase with time, meaning the hydroxide ions take longer to diffuse through and consequently the rate of glass dissolution will decrease. For this scenario, the 2-D diffusion model can be employed (6c):

$((1-\alpha) \ln (1-\alpha))+\alpha=r t$

Model (6c) describes the radial diffusion of species through a product layer of a long cylinder, and the thickness of this layer increases with time (unlike in previous cases where the product layer thickness is constant). The derivation of expression (6c) is presented elsewhere [26]. The reaction rate depends on factors such as the concentration of hydroxide ions and the rate of their diffusion through the product layer.

Models (6a-6c), or similar, have been applied previously for the dissolution of glass in alkaline solution [27-29]. The model which shows the best fit for our experimental data will indicate what was most likely to have been the rate-controlling factor of the glass fibre dissolution process. Figure 9 gives an example of model fitting for the case of glass fibre treatment in $3 \mathrm{~mol} / \mathrm{L} \mathrm{KOH}$ and $\mathrm{NaOH}$ at $75^{\circ} \mathrm{C}$ for 1 , 2,3 and $5 \mathrm{~h}$. The value of $\alpha$ was calculated from the fibre diameter reduction at each treatment time using Eq. (5), and these values were inserted into the models $(6 a-6 c)$ and plotted against treatment time t. A linear fit indicates that particular model best describes the glass fibre dissolution process. From Fig. $9 a, b$, it is clear that both the zero-order and shrinking cylinder models show an excellent fit, suggesting either the diffusion of $\mathrm{OH}^{-}$ions through the solution or the glass fibre etching itself (fibre 
(a)

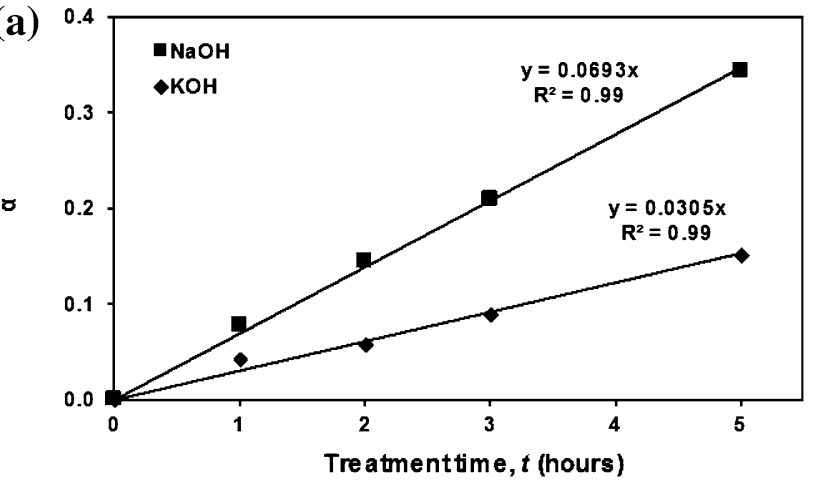

(c)

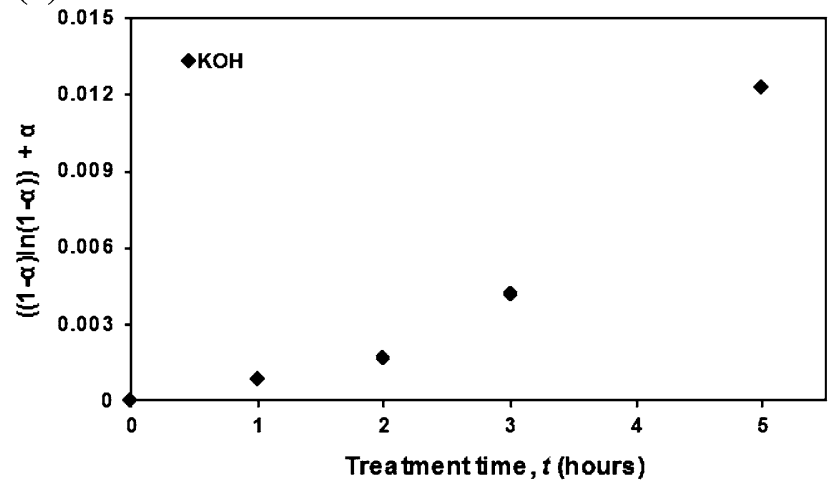

(b)

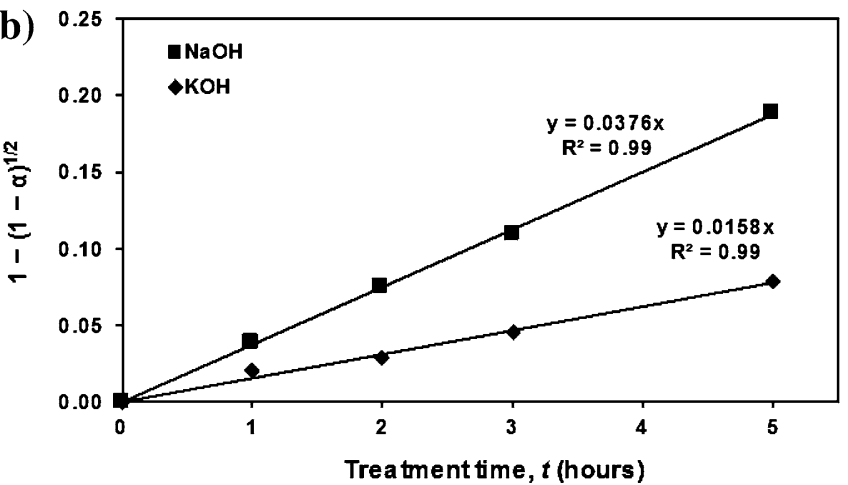

(d)

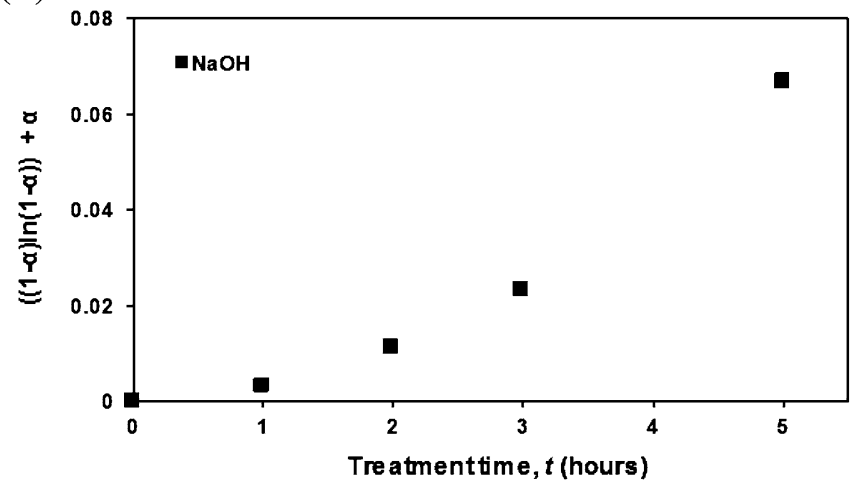

Figure 9 Fitting of kinetic data for $3 \mathrm{~mol} / \mathrm{L} \mathrm{KOH}$ and $\mathrm{NaOH}$ treatment of glass fibres at $75{ }^{\circ} \mathrm{C}$ in a zero-order model, $\mathbf{b}$ shrinking cylinder model, c 2-D diffusion model $(\mathrm{KOH})$, d 2-D diffusion model $(\mathrm{NaOH})$.

diameter reduction) could have been rate-controlling factors. The fact that the data fit well in both the zeroorder and shrinking cylinder models indicates the diffusion of hydroxide ions through the solution and the etching process could both have occurred at a similar rate.

Conversely, the nonlinearity for the plots (c) and (d) implies the 2-D diffusion model does not describe the reaction well and suggests the product layer present on the fibre surface was very thin or small amounts of product was built up on the surface since individual fibres were treated in alkaline solution. As a result, the diffusion of $\mathrm{OH}^{-}$ions through the product layer on the glass fibre was likely to have occurred at a rapid rate (in other words, not a ratedetermining step). This trend with model fitting was observed for all other alkaline treatment conditions. When the glass fibres reacted with the alkaline solution, a product layer was formed on the surface. This product layer was likely to have been comprised of sodium or potassium silicates, depending on whether $\mathrm{NaOH}$ or $\mathrm{KOH}$ was employed. Due to the extremely corrosive solution and the solubility of these silicates, it was likely that the product layer was constantly removed during the treatment duration; it was unlikely that the product layer increased in thickness to impede the glass dissolution reaction. During the acid rinsing procedure, any remnants of residue and product layer were effectively removed to allow an accurate measurement of the fibre diameter with SEM.

\section{Determination of reaction order and activation energy}

By conducting glass fibre treatments in various concentrations of alkaline solution, the reaction order with respect to $\mathrm{KOH}$ and $\mathrm{NaOH}$ and their associated rate constants can be determined. These parameters are related according to the following expression:

$r=k S\left[\mathrm{OH}^{-}\right]^{n}$

$\left[\mathrm{OH}^{-}\right]$is the initial concentration of alkaline solution in $\mathrm{mol} / \mathrm{L}$ and the superscript, $n$, is the reaction order with respect to $\mathrm{OH}^{-}$. $S$ is the initial glass surface area, and $k$ is the rate constant. The linear fit of our experimental data in models (6a) and (6b) according 
to 'Modelling of glass fibre dissolution in alkaline solution' section means the reaction rate $r$ (also denoted as initial reaction rate) from both the zeroorder and shrinking cylinder models can be used. Note the zero-order model refers to the reaction being zero order with respect to the glass. To determine the rate constant $k$ and the reaction order with respect to $\mathrm{KOH}$ and $\mathrm{NaOH}(n)$, the natural logarithm is taken of both sides of Eq. (7):

$\ln (r)=n \ln \left[\mathrm{OH}^{-}\right]+\ln (k S)$

Figure 10 shows how to obtain the reaction order through the use of Eq. (8); $-\ln (r)$ is plotted against $\ln \left[\mathrm{OH}^{-}\right]$, where $\left[\mathrm{OH}^{-}\right]$refers to the concentration of the $\mathrm{KOH}$ solution. The $r$ values for each $\mathrm{KOH}$ concentration are presented numerically in Table 2. Clearly, the reaction rate increases as a function of $\mathrm{KOH}$ solution concentration, and the data show a linear fit; the slope of the line gives the reaction order with respect to $\mathrm{KOH}(n)$, which are 0.43 and 0.49 for the zero-order and shrinking cylinder plots, respectively. The intercepts of the linear regression give the values of the product of rate constant and initial surface

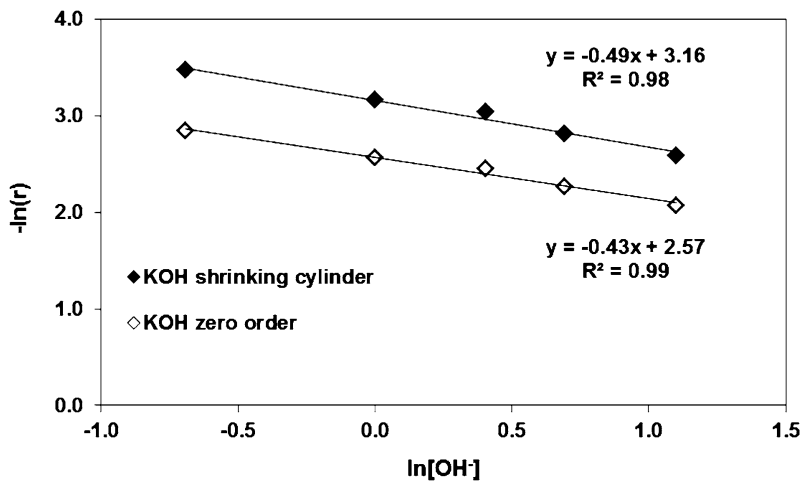

Figure 10 Plot to determine reaction order with respect to $\mathrm{KOH}$ through curve fitting. area of the fibre. Using the specific surface area of the glass fibre $\left(0.092 \mathrm{~m}^{2} / \mathrm{g}\right)$ in place of $S$, the value of the rate constant $k$ equals to $1.3 \times 10^{-4} \mathrm{~g} /\left(\mathrm{m}^{2} \mathrm{~s}\right)$ using the shrinking cylinder model and $2.4 \times 10^{-4} \mathrm{~g} /$ $\left(\mathrm{m}^{2} \mathrm{~s}\right)$ using the zero-order model.

Figure 11 shows the use of Eq. (8) to find the reaction order, this time, for $\mathrm{NaOH}$ (numerical data presented in Table 2). We can see from the gradients of the lines that the reaction order is 0.31 with the zero-order model and 0.44 for the shrinking cylinder model. The reaction order from the shrinking cylinder equation is slightly lower than what is observed for $\mathrm{KOH}$. The zero-order model for the reaction of $\mathrm{NaOH}$ with glass gives a significantly lower reaction order of 0.31 compared to $\mathrm{KOH}$, which suggests that increasing the concentration of $\mathrm{NaOH}$ did not increase the rate of diffusion of hydroxide ions to as much an extent as for $\mathrm{KOH}$. The diffusion of hydroxide ions from $\mathrm{NaOH}$ through the solution might already have been quite high due to the lack of interaction with neighbouring water molecules [24], which consequently resulted in a greater dissolution

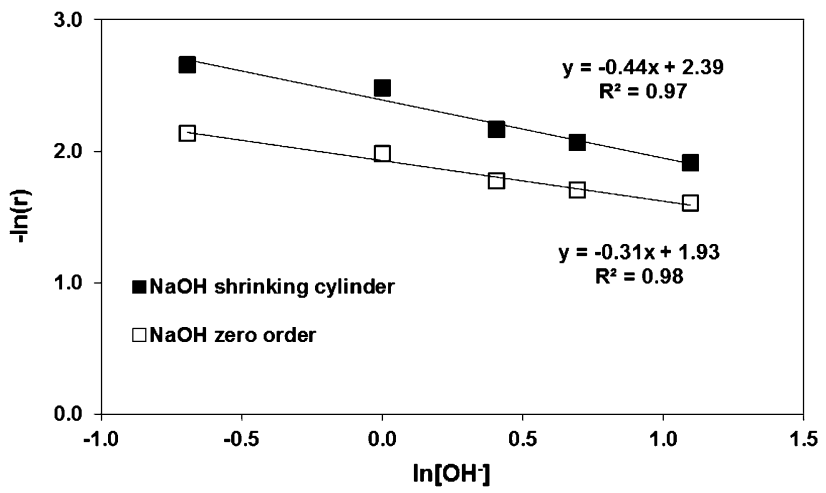

Figure 11 Plot to determine reaction order with respect to $\mathrm{NaOH}$ through curve fitting.

Table 2 Rate of reaction $(r)$ of glass fibre with $\mathrm{KOH}$ and $\mathrm{NaOH}$ solution at various concentrations and constant temperature $\left(95{ }^{\circ} \mathrm{C}\right)$

\begin{tabular}{|c|c|c|c|c|}
\hline \multirow[t]{3}{*}{ Concentration of alkaline solution $(\mathrm{mol} / \mathrm{L})$} & \multicolumn{4}{|c|}{ Reaction rate, $r(\% / h)$} \\
\hline & \multicolumn{2}{|l|}{$\mathrm{KOH}$} & \multicolumn{2}{|l|}{$\mathrm{NaOH}$} \\
\hline & Zero order & Shrinking cylinder & Zero order & Shrinking cylinder \\
\hline 0.5 & 5.8 & 3.1 & 11.9 & 7.0 \\
\hline 1.0 & 7.7 & 4.2 & 13.8 & 8.4 \\
\hline 1.5 & 8.6 & 4.8 & 17.0 & 11.4 \\
\hline 2.0 & 10.4 & 6.0 & 18.2 & 12.6 \\
\hline 3.0 & 12.6 & 7.5 & 20.1 & 14.7 \\
\hline
\end{tabular}


rate of the glass compared to when $\mathrm{KOH}$ was used. Reaction orders of $0.43-0.49$ for $\mathrm{KOH}$ and $0.31-0.44$ for $\mathrm{NaOH}$ are fairly consistent with the value reported by Jendoubi et al. [30] of 0.47 when reacting silica sand and aqueous $\mathrm{NaOH}$. From these calculated reaction orders, it can be said that doubling the concentration of alkaline solution leads to an increase in reaction rate by around 1.38 for $\mathrm{KOH}$ and 1.30 for $\mathrm{NaOH}$. Furthermore, the rate constant for glass fibre treatment in $\mathrm{NaOH}$ at $95{ }^{\circ} \mathrm{C}$ is found to be $2.7 \times 10^{-4} \mathrm{~g} /\left(\mathrm{m}^{2} \mathrm{~s}\right)$ from the shrinking cylinder model and $4.3 \times 10^{-4} \mathrm{~g} /\left(\mathrm{m}^{2} \mathrm{~s}\right)$ from the zero-order model. Note the prediction of the reaction rate at any alkaline solution concentration can be achieved using the correlating equations given in Figs. 10 and 11 or alternatively the non-logarithm form in Eq. (7) with using $k S$.

As well as determining the reaction order for $\mathrm{KOH}$ and $\mathrm{NaOH}$ in the glass fibre dissolution process, the activation energy $\left(E_{\mathrm{a}}\right)$ may be calculated through the application of the Arrhenius equation:

$k=A \mathrm{e}^{\frac{-E_{\mathrm{a}}}{R T}}$

$A$ is the pre-exponential factor, $R$ is the gas constant $[8.314 \mathrm{~J} /(\mathrm{mol} \mathrm{K})]$ and $T$ is the absolute temperature. The $E_{\mathrm{a}}$ value can be calculated through graphing similar to what is shown in Figs. 10 and 11. Firstly, the natural logarithm is taken of both sides of Eq. (9) to convert it to Eq. (10):

$\ln (k)=\left(\frac{-E_{\mathrm{a}}}{R}\right) \frac{1}{T}+\ln (A)$

The rate constant, $k$, can be calculated for each temperature change using Eq. (7). The value of $k$ after treatment of glass fibres in $3 \mathrm{~mol} / \mathrm{L} \mathrm{KOH}$ and $\mathrm{NaOH}$ solutions at different temperatures is presented in Table 3. To obtain the activation energy, $\ln (k)$ is plotted against the reciprocal of $T\left(\times 10^{3}\right)$, as shown in Fig. 12 with the case of glass fibre treatment in $\mathrm{KOH}$. We have seen in Fig. 8 that as the temperature of the alkaline solution increases, the rate of fibre diameter reduction increases in an exponential fashion. Hence, taking the natural logarithm of the rate constant and plotting against the reciprocal of solution temperature results in a straight line, as shown in Fig. 12, when using $k$ values from both the zero-order and shrinking cylinder models. Because $1 / T$ is already multiplied by $10^{3}$, the slope of the line $\left(-E_{\mathrm{a}} /\right.$ $R)$ is simply multiplied by the gas constant $R$ to obtain $E_{\mathrm{a}}$ directly in $\mathrm{kJ} / \mathrm{mol}$. The activation energy for the reaction of glass fibre with $\mathrm{KOH}$ solution is therefore calculated as $72 \mathrm{~kJ} / \mathrm{mol}$ (using $k$ values from the zero-order model) and $79 \mathrm{~kJ} / \mathrm{mol}$ (using $k$ values from the shrinking cylinder model). It is apparent that the activation energies are similar when rate constants are used from both models. The values are comparable with those in the literature concerning the reaction of bulk silicate glass or powder with alkaline solution [22, 28, 31].

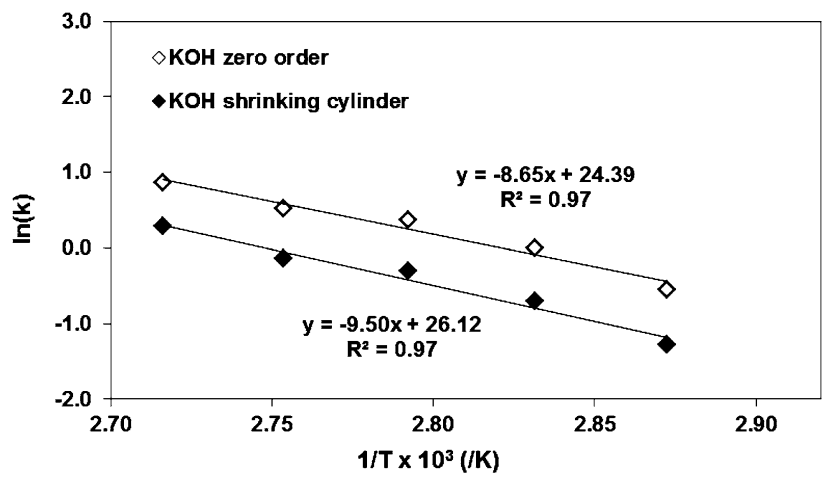

Figure 12 Plot to determine activation energy for glass fibre reaction with $\mathrm{KOH}$ through curve fitting.

Table 3 Rate constant $(k)$ of glass fibre reaction with $\mathrm{KOH}$ and $\mathrm{NaOH}$ solutions at various temperatures and constant concentration $(3 \mathrm{~mol} / \mathrm{L})$

\begin{tabular}{|c|c|c|c|c|}
\hline \multirow[t]{3}{*}{ Temperature of alkaline solution $\left({ }^{\circ} \mathrm{C}\right)$} & \multicolumn{4}{|c|}{ Rate constant, $k\left[\times 10^{-4} \mathrm{~g} /\left(\mathrm{m}^{2} \mathrm{~s}\right)\right]$} \\
\hline & \multicolumn{2}{|l|}{$\mathrm{KOH}$} & \multicolumn{2}{|l|}{$\mathrm{NaOH}$} \\
\hline & Zero order & Shrinking cylinder & Zero order & Shrinking cylinder \\
\hline 75 & 0.6 & 0.3 & 1.5 & 0.7 \\
\hline 80 & 1.0 & 0.5 & 2.0 & 0.9 \\
\hline 85 & 1.4 & 0.7 & 2.9 & 1.5 \\
\hline 90 & 1.7 & 0.9 & 3.6 & 2.0 \\
\hline 95 & 2.4 & 1.3 & 4.3 & 2.7 \\
\hline
\end{tabular}


The plots of $\ln (k)$ against $1 / T\left(\times 10^{3}\right)$ for glass fibres treated in $3 \mathrm{~mol} / \mathrm{L} \mathrm{NaOH}$ and the raw numerical data are displayed in Fig. 13 and Table 3, respectively. Similar to $\mathrm{KOH}$, the plots are linear in nature and the gradients are multiplied by the gas constant to give activation energies of $58 \mathrm{~kJ} / \mathrm{mol}$ (using $k$ values from the zero-order model) and $74 \mathrm{~kJ} / \mathrm{mol}$ (using $k$ values from the shrinking cylinder model). On this occasion, there is a disparity in the calculated activation energy when using rate constants from the two models. The activation energy of the glass fibre reaction with $\mathrm{NaOH}$ from the shrinking cylinder model is similar to the value given for $\mathrm{KOH}$ from the same model, suggesting the initiation of the glass fibre etching process required similar energy regardless of whether $\mathrm{KOH}$ or $\mathrm{NaOH}$ was employed as the alkaline solution. Because the shrinking cylinder model defines the glass fibre etching mechanism, which results in fibre diameter reduction, it makes sense to use the activation energy obtained from this model as the activation energy of the reaction of glass fibre with alkaline solution. The activation energy given from the zero-order model for glass fibre treatment in $\mathrm{NaOH}$ is significantly lower than for $\mathrm{KOH}$; this suggests less energy was required for the diffusion of hydroxide ions through the solution if they originated from $\mathrm{NaOH}$. This is unusual considering both $\mathrm{KOH}$ and $\mathrm{NaOH}$ should effectively have dissociated into metal cations and hydroxide anions in solution; in fact, the dissociation constant for $\mathrm{KOH}$ was expected to have been slightly higher. This shows how the nature of the metal cation could have played a significant role in the diffusion of the hydroxide ions through the solution, which consequently had an effect on the dissolution rate of

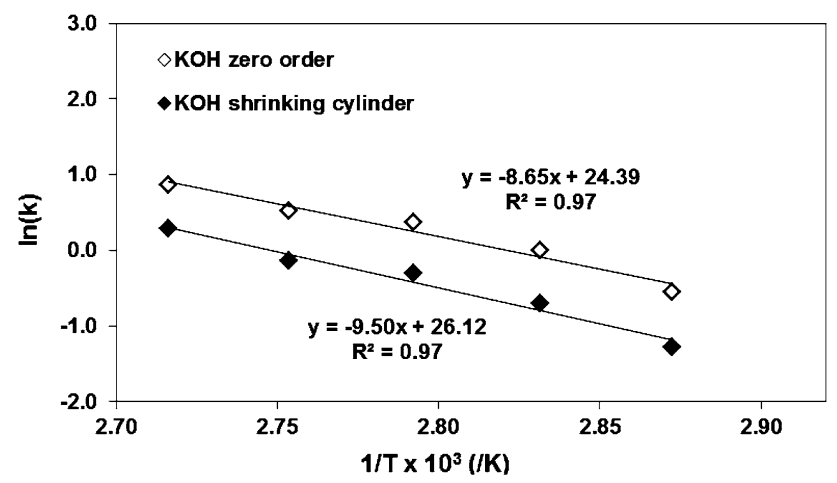

Figure 13 Plot to determine activation energy for glass fibre reaction with $\mathrm{NaOH}$ through curve fitting. the glass fibres. Overall, the activation energies obtained from the reaction of glass fibre with $\mathrm{KOH}$ and $\mathrm{NaOH}$ solutions are similar to those reported in previous studies [22, 28, 31], although the value of $28 \mathrm{~kJ} / \mathrm{mol}$ for the reaction of diatomite with $\mathrm{NaOH}$ is lower [29], and $159 \mathrm{~kJ} / \mathrm{mol}$, given by Molchanov and Prikhidko [18] for the dissolution of quartz glass in $\mathrm{NaOH}$ solution, is significantly high; this comes as no surprise given that quartz is a crystalline form of silica and is therefore more stable towards hydroxide attack.

\section{Conclusions}

The dissolution behaviour of individual E-glass fibres in high-temperature and corrosive alkaline environments was studied. Our kinetic data show the diameter reduction in fibres after $\mathrm{KOH}$ or $\mathrm{NaOH}$ treatment increases fairly linearly with time. $\mathrm{NaOH}$ was more reactive than $\mathrm{KOH}$ with E-glass fibres; at times we found $\mathrm{NaOH}$ to etch glass fibres up to twice the magnitude of $\mathrm{KOH}$. Increasing the concentration and temperature of the alkaline solution resulted in an increase in the reaction rate. Given the alkaline solutions were highly concentrated and prepared at elevated temperatures, it is believed the hydrolysis of the glass network by $\mathrm{OH}^{-}$ions was a key mechanism in the glass fibre dissolution process. The fact that our data fit best in the shrinking cylinder and zero-order models indicates the etching of the glass fibre and/or the diffusion of the hydroxide ions through the solution could have been rate-controlling factors. The generally poor agreement with the 2-D diffusion model implies the diffusion of the $\mathrm{OH}^{-}$ions through the product layer on the glass surface was unlikely to have been a rate-determining step. The reaction rates and rate constants obtained from the zero-order and shrinking cylinder models were used in the determination of the reaction order with respect to the alkaline solution and the activation energy. The reaction order was found to be $0.43-0.49$ for $\mathrm{KOH}$ and $0.31-0.44$ for $\mathrm{NaOH}$. It was evident, particularly with using the rate constants from the zero-order equation, that the activation energy for the reaction of E-glass fibre with $\mathrm{NaOH}(58 \mathrm{~kJ} / \mathrm{mol})$ was significantly lower than for $\mathrm{KOH}(72 \mathrm{~kJ} / \mathrm{mol})$; this suggests that the diffusion of the hydroxide ions through the solution required less energy if $\mathrm{NaOH}$ was employed as the corrosive medium. The fact that hydroxide 
ions from $\mathrm{NaOH}$ diffused more easily through the solution could have explained the greater dissolution of the glass fibres. In addition, the similar values of activation energy from the shrinking cylinder model for glass fibre dissolution in $\mathrm{KOH}(79 \mathrm{~kJ} / \mathrm{mol})$ and $\mathrm{NaOH}(74 \mathrm{~kJ} / \mathrm{mol})$ imply that once the hydroxide ions were at the glass surface, the energy needed to initiate the etching procedure was the same regardless of the nature of alkaline solution used. In addition, the rate constant for the reaction of glass fibre with alkaline solution at $95{ }^{\circ} \mathrm{C}$ was found to be between $1.3 \times 10^{-4}$ and $4.3 \times 10^{-4} \mathrm{~g} /\left(\mathrm{m}^{2} \mathrm{~s}\right)$.

\section{Acknowledgements}

The authors would like to thank Owens Corning for providing the glass fibres used in this study. The authors also gratefully acknowledge the University of Strathclyde for funding this research project. Gratitude is extended to the Advanced Materials Research Laboratory (AMRL) for their assistance in SEM analysis and members of the Advanced Composites Group for their help and support.

Open Access This article is distributed under the terms of the Creative Commons Attribution 4.0 International License (http://creativecommons.org/ licenses/by/4.0/), which permits unrestricted use, distribution, and reproduction in any medium, provided you give appropriate credit to the original author(s) and the source, provide a link to the Creative Commons license, and indicate if changes were made.

\section{References}

[1] Job S, Leeke G, Mativenga PT, Oliveux G, Pickering S, Shuaib NA (2016) Composites recycling: where are we now? Report for Composites UK Ltd. https://compositesuk. co.uk/system/files/documents/Recycling\%20Report\%202016. pdf

[2] Owens Corning Investor Presentation (slide 22). http://s1. q4cdn.com/942908807/files/doc_presentations/2015/Q3/Q3Presentation-v9.pdf

[3] Job S (2013) Recycling glass fibre reinforced compositeshistory and progress. Reinf Plast 57(5):19-23

[4] Albers H, Greiner S, Seifertand H, Kühne U (2009) Recycling of wind turbine rotor blades-fact or fiction? DEWI Mag 34:32-38
[5] Oliveux G, Dandy L, Leeke G (2015) Current status of recycling of fibre reinforced polymers: review of technologies, reuse and resulting properties. Prog Mater Sci 72:61-99

[6] Pickering SJ (2006) Recycling technologies for thermoset composite materials - current status. Compos A Appl Sci Manuf 37:1206-1215

[7] Thomason JL, Yang L, Meier R (2014) The properties of glass fibres after conditioning at composite recycling temperatures. Compos A Appl Sci Manuf 61:201-208

[8] Jenkins PG, Yang L, Liggat JJ, Thomason JL (2015) Investigation of the strength loss of glass fibre after thermal conditioning. J Mater Sci 50:1050-1057. doi:10.1007/ s10853-014-8661-x

[9] Feih S, Boiocchi E, Mathys G, Gibson AG, Mouritz AP (2011) Mechanical properties of thermally-treated and recycled glass fibres. Compos B Eng 42(3):350-358

[10] Nagel U, Yang L, Kao CC, Thomason JL (2016) Effects of thermal recycling temperatures on the reinforcement potential of glass fibers. Polym Compos 36(9):1-9

[11] Bashir ST, Yang L, Anderson R, Tang PL, Liggat JJ, Thomason JL (2017) A simple chemical approach to regenerating the strength of thermally damaged glass fibre. Compos A Appl Sci Manuf 102:76-87

[12] Yang L, Sáez ER, Nagel U, Thomason JL (2015) Can thermally degraded glass fibre be regenerated for closed-loop recycling of thermosetting composites? Compos A Appl Sci Manuf 72:167-174

[13] Spierings GACM (1993) Wet chemical etching of silicate glasses in hydrofluoric acid based solutions. J Mater Sci 28(23):6261-6273. doi:10.1007/BF01352182

[14] Thomason J, Nagel U, Yang L, Sáez E (2016) Regenerating the strength of thermally recycled glass fibres using hot sodium hydroxide. Compos A Appl Sci Manuf 87:220-227

[15] Sáez E (2016) Regenerating the strength of thermally recycled glass fibres using chemical treatments. PhD thesis, University of Strathclyde

[16] Kouassi S, Andji J, Bonnet J, Rossignol S (2010) Dissolution of waste glasses in high alkaline solutions. Ceram Silik 54(3):235-240

[17] Smith R, Corbin P (1949) Attack of glasses by alkaline solutions. J Am Ceram Soc 32(6):195-198

[18] Molchanov VS, Prikhidko NE (1995) Corrosion of silicate glasses by alkaline solutions. Bull Acad Sci USSR Div Chem Sci 6:1179-1184

[19] Scheffler C, Förster T, Mäder E, Heinrich G, Hempel S, Mechtcherine V (2009) Aging of alkali-resistant glass and basalt fibers in alkaline solutions: evaluation of the failure stress by Weibull distribution function. J Non-Cryst Solids 355(52-54):2588-2595 
[20] Yang L, Thomason JL (2013) Effect of silane coupling agent on mechanical performance of glass fibre. J Mater Sci 48:1947-1954. doi:10.1007/s10853-012-6960-7

[21] Wei B, Cao H, Song S (2010) Tensile behavior contrast of basalt and glass fibers after chemical treatment. Mater Des 31:4244-4250

[22] Molchanov VS, Prikhidko NE (1958) Corrosion of silicate glasses by alkaline solutions Communication 4. Corrosion of glasses by solutions of various hydroxides. Bull Acad Sci USSR Div Chem Sci 7(8):893-897

[23] Hooley J (1961) The kinetics of the reaction of silica with group I hydroxides. Can J Chem 39(6):1221-1230

[24] Chen B, Ivanov I, Park J, Parrinello M, Klein M (2002) Solvation structure and mobility mechanism of $\mathrm{OH}^{-}$: a CarParrinello molecular dynamics investigation of alkaline solutions. J Phys Chem B 106(46):12006-12016

[25] Gin S, Jollivet P, Fournier M, Berthon C, Wang Z, Mitroshkov A, Zhu Z, Ryan J (2015) The fate of silicon during glass corrosion under alkaline conditions: a mechanistic and kinetic study with the International Simple Glass. Geochim Cosmochim Acta 151:68-85
[26] Khawam A, Flanagan D (2006) Solid-state kinetic models: basics and mathematical fundamentals. J Phys Chem B 110(35):17315-17328

[27] Brouwers HJH, van Eijk RJ (2002) Reactivity of fly ash: extension and application of a shrinking core model. Concr Sci Eng 4:106-113

[28] Fernandez J, Renedo M, Pesquera A, Irabien J (2002) Kinetic study of the hydrothermal reaction of fly ash with $\mathrm{ca}(\mathrm{oh}) 2$ in the preparation of desulfurant sorbents. Chem Eng Commun 189(3):310-321

[29] Du G, Lü G, He X (2013) apparent dissolution kinetics of diatomite in alkaline solution. Chin $J$ Chem Eng 21(7):736-741

[30] Jendoubi F, Mgaidi A, El Maaoui M (1997) Kinetics of the dissolution of silica in aqueous sodium hydroxide solutions at high pressure and temperature. Can J Chem Eng 75(4):721-727

[31] Greenberg S (1957) The depolymerization of silica in sodium hydroxide solutions. J Phys Chem 61(7):960-965 